
JNM
J Neurogastroenterol Motil, Vol. 22 No. 4 October, 2016
pISSN: 2093-0879 elSSN: 2093-0887
http://dx.doi.org/10.5056/jnm15203
Original Article

\title{
Granisetron Transdermal System for Treatment of Symptoms of Gastroparesis: A Prescription Registry Study
}

\author{
Deena Midani and Henry P Parkman* \\ Department of Medicine, Section of Gastroenterology, Temple University School of Medicine, Philadelphia, PA, USA
}

\begin{abstract}
Background/Aims
Serotonin receptor (eg, 5- $\mathrm{HT}_{3}$ ) antagonists are used to treat nausea and vomiting from a variety of causes. Granisetron transdermal system (GTS) is an appealing delivery system for patients with gastroparesis. To assess if GTS improves nausea and vomiting and other gastroparesis symptoms in patients with gastroparesis.
\end{abstract}

\section{Methods}

Patients with gastroparesis and symptoms of nausea and vomiting refractory to conventional treatment were treated with GTS. Symptoms of gastroparesis were assessed using a modified Gastroparesis Cardinal Symptom Index (GCSI). Following 2 weeks of treatment, patients were asked to assess their symptoms and indicate their therapeutic response using the Clinical Patient Grading Assessment Scale (CPGAS) reporting if symptoms of nausea and vomiting improved on a scale: $0=$ no change to $+7=$ completely better.

\section{Results}

Fifty-one patients received GTS by prescription: average age was $40 \pm 17$ years, 44 female, 11 diabetics, $23 \pm 20 \%$ retention at 4 hours on gastric emptying scintigraphy. Thirty-nine of the 51 (76\%) patients stated improvement with GTS. There was significant improvement in nausea and vomiting as assessed with CPGAS at 2 weeks $(2.28 \pm 2.53 ; P<0.05)$. Symptoms of nausea and vomiting significantly improved. Other symptoms including postprandial fullness, loss of appetite, upper abdominal pain, and early satiety improved. Side effects reported included redness at the site of the patch in 7 patients, pruritus in 5, and constipation in 5.

\section{Conclusions}

GTS was moderately effective in reducing nausea and/or vomiting in $76 \%$ of gastroparesis patients. In addition to nausea and vomiting, symptoms of postprandial fullness, loss of appetite, upper abdominal pain, and early satiety also improved.

(J Neurogastroenterol Motil 2016;22:650-655)

Key Words

Diabetic gastroparesis; Gastroparesis; Nausea; Vomiting

Received: December 29, 2015 Revised: March 9, 2016 Accepted: April 9, 2016

(a) This is an Open Access article distributed under the terms of the Creative Commons Attribution Non-Commercial License (http://creativecommons. org/licenses/by-nc/4.0) which permits unrestricted non-commercial use, distribution, and reproduction in any medium, provided the original work is properly cited.

*Correspondence: Henry P Parkman, MD

GI Section, Temple University Hospital, 3401 N. Broad Street, Philadelphia, PA 19140, USA

Tel: +1-215-707-3431, Fax: +1-215-707-2684, E-mail: henry.parkman@temple.edu 


\section{Introduction}

Gastroparesis is a chronic, symptomatic disorder characterized by delayed gastric emptying. ${ }^{1}$ Symptoms of gastroparesis includes nausea, vomiting, early satiety, postprandial fullness, and upper abdominal pain. Nausea and vomiting, the classic symptoms of gastroparesis, contribute to the decreased quality of life seen in patients with this disorder. ${ }^{2}$ Treatment of gastroparesis often involves prokinetic agents that enhance gastric emptying and symptom modulators that reduce symptoms.

Serotonin receptor (eg, 5- $\mathrm{HT}_{3}$ ) antagonists, such as ondansetron and granisetron, are approved for chemotherapy-induced, radiation induced, and postoperative nausea and vomiting., ${ }^{3,4}$ These agents are also being used to treat nausea and vomiting due to a variety of other causes, such as gastroparesis. Little data are available on the use of $5-\mathrm{HT}_{3}$ antagonists for reduction of nausea and vomiting in gastroparesis. Oral treatment with medications can be problematic in some patients with gastroparesis, as nausea and vomiting can prevent oral ingestion and delayed gastric emptying can delay absorption, resulting in unpredictable efficacy. The granisetron transdermal system (GTS; Sancuso) releases $3.1 \mathrm{mg}$ of granisetron per 24 hours for up to 7 days and is approved in the USA for prevention of nausea and vomiting in patients receiving moderately or highly emetogenic chemotherapy for up to five consecutive days. The transdermal route is a potentially appealing drug delivery system for patients with gastroparesis. This delivery system is not dependant on gastric emptying and may be used in patients with significant vomiting precluding oral intake. We have previously reported results with GTS in decreasing nausea and vomiting in gastroparesis. ${ }^{5}$ Patients with gastroparesis have a variety of symptoms; the effects of GTS on other symptoms of gastroparesis are not known.

The aim of this study was to assess if treatment of patients with gastroparesis with GTS (Sancuso) improves not only nausea and vomiting, but also other associated symptoms of gastroparesis.

\section{Materials and Methods}

This clinical protocol was conducted prospectively at Temple University Hospital in patients with refractory gastroparesis under a protocol, which had been approved at our institution by our Institutional Review Board.

Patients being seen at the Temple University Hospital Gastroenterology section for gastroparesis and who had symptoms of nausea and vomiting refractory to conventional antiemetic treatment participated in this open-label prescription registry study. Patients were identified as having gastroparesis if they had classical symptoms of gastroparesis, particularly nausea and vomiting, with previous negative upper endoscopy and a prior delayed gastric emptying test. These patients were refractory in that they had not responded or had side effects to conventional treatment for gastroparesis including antiemetic agents (eg, ondansetron, promethazine, and prochlorperazine) and promotility agents (eg, metoclopramide and domperidone). During an outpatient office visit, potential treatment options were discussed for their persistent symptoms, including the use of GTS. Patients could continue medications that gave partial responses including antiemetic agents (eg, promethazine and prochlorperazine) and/or promotility agents (eg, metoclopramide and domperidone). Consenting patients were given a prescription for GTS with directions to reapply one patch every seven days on the upper arm. Patients could continue their current antiemetic and/or prokinetic medications at their regular dosage.

Patients completed questionnaires to acquire data prior to treatment with the granisetron transdermal system and at subsequent visits. Baseline was defined as the 2 -week period before treatment. The patient assessment of gastrointestinal symptoms (PAGI-SYM) questionnaires included the gastroparesis cardinal symptom index (GCSI) scores as well as additional questions concerning abdominal pain, constipation, and diarrhea. ${ }^{6,7}$ The GCSI evaluates the severity of nine symptoms over the past 2 weeks using a Likert scale from 0 (none) to 5 (very severe). ${ }^{7}$ The symptoms include nausea, retching, vomiting, stomach fullness, inability to finish a standard meal, feeling excessively full after meals, loss of appetite, bloating, and a visibly larger stomach. Questions were also posed about the presence of upper abdominal pain, upper abdominal discomfort, constipation, and diarrhea with similar scoring to the GCSI symptoms.

The results of their clinical tests previously obtained were recorded including upper endoscopy and gastric emptying scintigraphy (GES). In most patients, gastric emptying was assessed with the 4-hour Egg Beaters (ConAgra Foods, Omaha, NE, USA) and GES (Abell, Tougas). Delayed gastric emptying is defined as $>60 \%$ retention at 2 hours and/or $>10 \%$ retention at 4 hours. ${ }^{8,9}$

Following 2 weeks of treatment with GTS, patients were asked to assess their therapeutic response. For assessment of global clinical response, the following question was utilized, "In thinking about the past 2 weeks, how would you say your nausea and vomiting symptoms have been compared to before you started the Sancuso treatment?" Responses were improved, no change, worsened. Patients were then asked to quantify their therapeutic response to 
nausea and vomiting using the clinical patient grading assessment scale (CPGAS). ${ }^{10}$ Patients selected a number over the range $(+7$ $=$ completely better, $0=$ no change, and $-7=$ very much worse) that best answered the question. This response scale has been used in other studies assessing outcomes in gastroparesis. ${ }^{5,10}$ Patients also completed modified PAGI-SYM questionnaires including the GCSI scores as well as additional questions concerning abdominal pain, constipation, and diarrhea. Finally, patients were asked if they experienced side effects with treatment, and if so, what they were.

\section{Statistical Methods}

Data was compiled in a Microsoft Excel database. Results are expressed as percentage of patients, or mean $\pm \mathrm{SD}$ or mean \pm SEM where appropriate. Analyses were performed using paired Student's $t$ test, ANOVA, Pearson correlation coefficient, and Chisquared where appropriate. A $P$-value of $<0.05$ was considered statistically significant.

\section{Results}

\section{Patients}

There were 118 gastroparesis patients who were given prescriptions for Sancuso (granisetron transdermal system) for treatment of their refractory nausea/vomiting in this open-label prescription registry study. In general, patients had not responded or had side effects to the prokinetics agent metoclopramide and the antiemetic agents prochlorperazine and ondansetron. Of these, 51 patients started treatment with Sancuso by prescription. The reasons given for not obtaining the GTS included: insurance did not cover the medication, medication too expensive (high co-pay or limited insurance coverage), other treatments started to work prior to starting treatment with the GTS. Table 1 shows the demographic information on the 51 patients undergoing treatment with granisetron transdermal patch. The average age was $41.1 \pm 16.3$ years. There were 44 females and 7 males. The etiologies of the gastroparesis were diabetic in 13 patients, idiopathic in 35 patients, and 3 had other types of gastroparesis. The delay in gastric emptying averaged $25.5 \pm 21.0 \%$ retention at 4 hours on GES (normal $<10 \%)$.

\section{Response to Treatment}

Thirty-nine of the $51(76 \%)$ patients reported improvement with the GTS. Overall, there was significant improvement in symptoms of nausea and vomiting as assessed with the CPGAS at 2 weeks $(2.28 \pm 2.53, P<0.05)$. A score of 3 indicates the patient being "somewhat better".

Using the GCSI to assess the severity of symptoms, many of the gastroparesis symptoms improved with treatment (Table 2). The symptom of nausea improved most significantly, with a decrease in the symptom severity score of 1.18 indicating a drop in a full category on the symptom severity scale. Vomiting also improved with a decrease in symptom score of 0.91 . Other symptoms that particularly improved were postprandial fullness (0.91) and loss of appetite (0.90). There were also improvements seen in early satiety (0.71) and upper abdominal pain (0.78). Other symptoms also significantly improved but to lesser extent; these included stomach fullness (0.62), abdominal distension (0.56), bloating (0.69), and upper abdominal discomfort (0.73). Diarrhea also improved (0.95).

Factors that might impact the response of the GTS are shown in Table 3. There were no apparent effects of gender, age, or etiology of gastroparesis on the favorable effect of GTS on nausea and vomiting. There were trends for patients with a higher body mass index $(\mathrm{BMI})\left(>30 \mathrm{~kg} / \mathrm{m}^{2}\right)$ to be associated with a better clinical response than patients with a normal or low $\mathrm{BMI}\left(<30 \mathrm{~kg} / \mathrm{m}^{2}\right)(P=0.082)$. Patients with a severe delay in gastric emptying $(>30 \%$ gastric retention) tended to be associated with less favorable response than those with not as severe delay in gastric emptying $(P=0.065)$.

Table 1. Baseline Characteristics in Gastroparesis Patients Treated with Granisetron Transdermal System for Nausea and/or Vomiting

\begin{tabular}{lc}
\hline \multicolumn{1}{c}{ Number of patients } & $\mathrm{n}=51$ \\
\hline Age (mean $\pm \mathrm{SD}, \mathrm{yr})$ & $41.1 \pm 16.3$ \\
Female/male $(\mathrm{n})$ & $44(86.3 \%) / 7(13.7 \%)$ \\
Etiology $(\mathrm{n})$ & $13(25.5 \%)$ \\
Diabetic & $35(68.6 \%)$ \\
Idiopathic & 2 \\
Other; post fundoplication & 1 \\
Mixed connective tissue disorder & \\
Race (n) & $44(86.3 \%)$ \\
Caucasian & $5(9.8 \%)$ \\
African American & $2(3.9 \%)$ \\
Other & $27.3 \pm 10.1$ \\
Body mass index $\left(\mathrm{kg} / \mathrm{m}^{2}\right)$ & \\
Gastric emptying & \\
$\%$ retention $2 \mathrm{hr}$ & $61.6 \pm 20.7$ \\
$\%$ retention $4 \mathrm{hr}$ & $25.5 \pm 21.0$ \\
Main symptom ${ }^{\mathrm{b}}(\mathrm{n})$ & \\
Nausea & $38(74.5 \%)$ \\
Vomiting & $9(17.6 \%)$ \\
Abdominal pain & $5(10.0 \%)$ \\
\hline
\end{tabular}

${ }^{\mathrm{a}}$ Gastric emptying using 4-hour scintigraphy was obtained in 44 patients. ${ }^{\mathrm{b}} \mathrm{Some}$ patients reported more than one main symptom. 
Table 2. Symptom Severity Response to Treatment with Granisetron Transdermal Patch

\begin{tabular}{|c|c|c|c|c|}
\hline Symptom & Baseline $^{a}$ & Treatment $^{\mathrm{a}}$ & Improvement in severity ${ }^{\mathrm{b}}$ & $P$-value \\
\hline Nausea & $4.17 \pm 0.73$ & $2.99 \pm 1.12$ & 1.18 & $<0.001$ \\
\hline Retching & $2.60 \pm 1.77$ & $2.00 \pm 1.67$ & 0.60 & 0.058 \\
\hline Vomiting & $2.57 \pm 1.66$ & $1.66 \pm 1.62$ & 0.91 & $<0.001$ \\
\hline Early satiety & $4.12 \pm 1.06$ & $3.41 \pm 1.36$ & 0.71 & 0.001 \\
\hline Postprandial fullness & $4.08 \pm 1.19$ & $3.17 \pm 1.42$ & 0.91 & $<0.001$ \\
\hline Stomach fullness & $3.68 \pm 1.06$ & $3.06 \pm 1.29$ & 0.62 & 0.001 \\
\hline Loss of appetite & $3.80 \pm 1.03$ & $2.90 \pm 1.47$ & 0.90 & $<0.001$ \\
\hline Stomach visibly larger & $2.50 \pm 1.66$ & $1.94 \pm 1.73$ & 0.56 & 0.029 \\
\hline Bloating & $3.02 \pm 1.51$ & $2.33 \pm 1.58$ & 0.69 & 0.001 \\
\hline Upper abdominal pain & $3.19 \pm 1.51$ & $2.41 \pm 1.54$ & 0.78 & 0.004 \\
\hline Upper abdominal discomfort & $3.32 \pm 1.49$ & $2.59 \pm 1.46$ & 0.73 & 0.002 \\
\hline Constipation & $2.56 \pm 1.91$ & $2.27 \pm 1.74$ & 0.29 & 0.330 \\
\hline Diarrhea & $1.85 \pm 1.83$ & $0.90 \pm 1.44$ & 0.95 & 0.001 \\
\hline
\end{tabular}

${ }^{a}$ Results expressed as mean $\pm \mathrm{SD}$.

${ }^{\mathrm{b}}$ Improvement in severity is the difference in the mean treatment score and the main baseline score.

Table 3. Factors That May Impact on the Response to Granisetron Transdermal Patch

\begin{tabular}{|c|c|c|c|}
\hline Factor & $\begin{array}{c}\text { CPGAS } \\
(\text { mean } \pm \mathrm{SD})\end{array}$ & $\mathrm{n}$ & $P$-value \\
\hline All Gastroparesis Patients & $2.28 \pm 2.53$ & 51 & \\
\hline \multicolumn{4}{|l|}{ Age } \\
\hline$<40 \mathrm{yr}$ & $2.18 \pm 2.88$ & 23 & 0.811 \\
\hline$>40 \mathrm{yr}$ & $2.35 \pm 2.28$ & 28 & \\
\hline \multicolumn{4}{|l|}{ Sex } \\
\hline Female & $2.19 \pm 2.46$ & 44 & 0.514 \\
\hline Male & $2.86 \pm 3.13$ & 7 & \\
\hline \multicolumn{4}{|l|}{ Etiology of gastroparesis $^{\mathrm{a}}$} \\
\hline Diabetic gastroparesis & $2.46 \pm 2.47$ & 13 & 0.554 \\
\hline Idiopathic gastroparesis & $1.97 \pm 2.55$ & 35 & \\
\hline \multicolumn{4}{|l|}{ Body mass index } \\
\hline$>30 \mathrm{~kg} / \mathrm{m}^{2}$ & $3.45 \pm 1.57$ & 12 & 0.082 \\
\hline $20-30 \mathrm{~kg} / \mathrm{m}^{2}$ & $1.90 \pm 2.77$ & 29 & \\
\hline$<20 \mathrm{~kg} / \mathrm{m}^{2}$ & $2.10 \pm 2.54$ & 10 & \\
\hline \multicolumn{4}{|l|}{ Gastric emptying $^{\mathrm{b}}$} \\
\hline$>30 \%$ retention at 4 hours & $1.15 \pm 3.13$ & 13 & 0.065 \\
\hline$<30 \%$ retention at 4 hours & $2.70 \pm 2.12$ & 30 & \\
\hline
\end{tabular}

${ }^{a}$ Some patients had gastroparesis that were postsurgical.

${ }^{b}$ Not all patients had complete 4 hour gastric emptying studies.

CPGAS, clinical patient grading assessment scale.

\section{Side Effects with Treatment}

Side effects reported were those anticipated: redness or rash at the site of the patch in 7 patients, local pruritus occurred in 5 patients, and constipation in 5 patients, headache in 1 , and heart racing in 1.

\section{Discussion}

In this open label prescription registry study, the GTS was effective in reducing nausea and/or vomiting in 76\% (39/51) of gastroparesis patients with refractory symptoms of nausea and vomiting. A prior study of ours also assessed the improvement in nausea and vomiting with the granisetron transdermal system. ${ }^{5}$ In this present study, we used the PAGI-SYM to capture the symptom severity of a variety of symptoms of gastroparesis, dyspepsia, and GERD. The 2 symptoms that showed particular improvement were nausea and vomiting. Interestingly, there were also improvements seen in symptoms of other related gastroparesis symptoms including postprandial fullness, loss of appetite, upper abdominal pain, and early satiety. Thus, the GTS may be effective for treating nausea and vomiting, and possibly other associated gastroparesis symptoms, in some refractory patients with gastroparesis.

The symptom most responsive to treatment was nausea, with a decrease in severity of 1.18 with treatment. Vomiting also significantly improved. This is not unexpected as the $5-\mathrm{HT}_{3}$ antagonists are helpful for nausea and vomiting in several conditions. ${ }^{3,4}$ Transdermal granisetron system is effective in the prevention of nausea and vomiting in patients with cancer who are receiving emetogenic chemotherapy. ${ }^{11,12}$ Perception of gut stimulation is also mediated by serotonergic (5-HT) receptors on intrinsic nerves and extrinsic sensory pathways that project to the nodose and dorsal root ganglia. ${ }^{13}$ $5-\mathrm{HT}_{3}$ mechanisms are involved in vomiting in a range of emetic 
stimulation; this has been primarily studied in chemotherapy-induced nausea and vomiting. $5-\mathrm{HT}_{3}$ receptors are on vagal afferent nerves projecting to the brainstem, as well as to the area postrema, nucleus tractus solitarius, and dorsal motor nucleus of the vagus, and may activate during emetic stimulation. ${ }^{13}$ Thus, there are $5-\mathrm{HT}_{3}$ receptors mediating pathways which transmit perception of nausea and vomiting from the gastrointestinal track.

Interestingly, this study in patients with symptomatic refractory gastroparesis demonstrated that there were improvements not only in nausea and vomiting, but also in other associated symptoms of gastroparesis including postprandial fullness, loss of appetite, upper abdominal pain, and early satiety. It may be that the improvement in the nausea and vomiting allows secondary improvement in the patient's perception of other symptoms such as early satiety since there is less nausea with eating. Alternatively, perhaps the $5-\mathrm{HT}_{3}$ antagonists have an effect on the neural pathways mediating these symptoms. 5- $\mathrm{HT}_{3}$ antagonists do not appear to alter gastric emptying. ${ }^{14}$ Other serotonergic receptors, particularly $5-\mathrm{HT}_{4}$ receptors, may be involved in the gastric accommodation response. ${ }^{15}$

This study examined factors that might impact the response to GTS in improving nausea and vomiting in patients with gastroparesis. Gender did not have an effect. Metoclopramide had been reported to have a more favorable effect in women than in men. ${ }^{16}$ Interestingly, obese patients, defined as BMI $>30 \mathrm{~kg} / \mathrm{m}^{2}$, tended to have a higher response rate to GTS than lower weight patients. Obese patients have been reported to have a higher response rate than other patients in other clinical trials which was suggested to be a higher placebo response rate. ${ }^{17}$ Patients with severely delayed gastric emptying had a lower response rate, which might be attributable to the need for prokinetic treatment in these patients to improve gastric emptying, rather than the antiemetic symptomatic effects provided by 5 - $\mathrm{HT}_{3}$ antagonists which do not promote gastric emptying. Etiology of gastroparesis did not have an effect on the favorable response of GTS on refractory symptoms as both patients with diabetic and idiopathic gastroparesis responded to GTS. These factors should be investigated in larger studies on the effect of granisetron in gastroparesis.

When used for chemotherapy-induced nausea and vomiting, the most common adverse reaction with GTP is constipation. ${ }^{4}$ The GTP may also cause some local skin irritation. In a previous clinical trial, application site reactions were reported which were generally mild in intensity and did not lead to discontinuation of use. ${ }^{12}$ The side effects seen in this study were expected and included redness at the site of the patch in 7 patients, pruritus in 5 , and constipation in 5 . One patient developed "heart racing". The medication was stopped prior to obtaining an electrocaridogram to see if there was corrected QT prolongation which can occur with 5- $\mathrm{HT}_{3}$ receptor antagonists.

This study evaluated the use of the GTS in clinical practice. It extends our prior study that showed an improvement in nausea and vomiting ${ }^{5}$ by increasing the number of patients studied with GTS, looking at other symptoms of gastroparesis, and evaluating some of the factors that might impact the response. There are limitations to this, as well as any, observational study. First, it was an open label study: patients knew they were getting an active agent. There is no comparative arm. Second, the sample size of 51 patients might explain the lack of significance in some of the factors that might impact treatment. Third, this study is from an academic institution to which refractory patients are being referred that have failed other treatment modalities. This study might underestimate the effective response as these patients treated had failed therapy with other agents. Fourth, it is a short term study assessing symptom response over several weeks. Gastroparesis is a chronic disorder for which patients need long term treatment. ${ }^{1}$ Parenthetically, many of the patients studied here were treated for longer periods of time after an initial favorable 2-week response and some had a sustained response to the granisetron patch. A randomized, double-blind placebocontrolled trial is needed to assess both the short term and long term effects of the GTS in treating patients with symptomatic refractory gastroparesis, especially with primary symptoms of nausea and vomiting. ${ }^{18}$

In conclusion, this open label prescription registry study shows that the GTS (Sancuso), was effective in reducing nausea and/or vomiting in 76\% (39/51) of gastroparesis patients with refractory symptoms of nausea and vomiting. Of interest, not only were nausea and vomiting improved, but there were also improvements seen in related symptoms including postprandial fullness, loss of appetite, upper abdominal pain, and early satiety. We conclude that the GTS may be an effective treatment for nausea and vomiting, and possibly for other related symptoms, in some gastroparesis patients.

Financial support: This study was supported by ProStrakan, Inc.

Conflicts of interest: Henry P Parkman has served as a consultant and on a product advisory board for ProStrakan, Inc, makers of Sancuso.

Author contributions: Deena Midani: data collection and critical revision of manuscript for important intellectual content; 
and Henry Parkman: study concept and design, study supervision, drafting of manuscript, and critical revision of the manuscript for important intellectual content.

\section{References}

1. Parkman HP, Hasler WL, Fisher RS. American Gastroenterological Association technical review on the diagnosis and treatment of gastroparesis. Gastroenterology 2004;127:1592-1622.

2. Cherian D, Parkman HP. Nausea and vomiting in diabetic and idiopathic gastroparesis. Neurogastroenterol Motil 2012;24:217-222, e103.

3. Keating GM, Duggan ST, Curran MP. Transdermal granisetron: a guide to its use in preventing nausea and vomiting induced by chemotherapy. CNS Drugs 2012;26:787-790.

4. Abramowicz M, Zuccotti G, Pflomm JM, et al. A granisetron patch (Sancuso). Med Lett Drugs Ther 2008;50:103-104.

5. Simmons K, Parkman HP. Granisetron transdermal system improves refractory nausea and vomiting in gastroparesis. Dig Dis Sci 2014;59:12311234.

6. Rentz AM, Kahrilas P, Stanghellini V, et al. Development and psychometric evaluation of the patient assessment of upper gastrointestinal symptom severity index (PAGI-SYM) in patients with upper gastrointestinal disorders. Qual Life Res 2004;13:1737-1749.

7. Revicki DA, Rentz AM, Dubois D, et al. Development and validation of a patient-assessed gastroparesis symptom severity measure: the Gastroparesis Cardinal Symptom Index. Aliment Pharmacol Ther 2003;18:141150.

8. Tougas G, Eaker EY, Abell TL, et al. Assessment of gastric emptying using a low fat meal: establishment of international control values. Am J Gastroenterol 2000;95:1456-1462.

9. Abell TL, Camilleri M, Donohoe K, et al. Consensus recommendations for gastric emptying scintigraphy: a joint report of the American
Neurogastroenterology and Motility Society and the Society of Nuclear Medicine. Am J Gastroenterology 2008;103:753-763.

10. Parkman HP, Mishra A, Jacobs M, et al. Clinical response and side effects of metoclopramide: associations with clinical, demographic, and pharmacogenetic parameters. J Clin Gastroenterol 2012;46:494-503.

11. Schulmeister L. Granisetron transdermal system: a new option to help prevent chemotherapy-induced nausea and vomiting. Clin J Oncol Nurs 2009;13:711-714.

12. . Boccia RV, Gordan LN, Clark G, Howell JD, Grunberg SM. Efficacy and tolerability of transdermal granisetron for the control of chemotherapy-induced nausea and vomiting associated with moderately and highly emetogenic multi-day chemotherapy: a randomized, double-blind, phase III study. Support Care Cancer 2011;19:1609-1617.

13. Hasler WL. Serotonin and the GI tract. Curr Gastroenterol Rep 2009;11:383-391.

14. Nielsen OH, Hvid-Jacobsen K, Lund P, Langoholz E. Gastric emptying and subjective symptoms of nausea: lack of effects of a 5-hydroxytryptamine-3 antagonist ondansetron on gastric emptying in patients with gastric stasis syndrome. Digestion 1990;46:89-96.

15. Tack J, Janssen P, Bisschops R, Vos R, Phillips T, Tougas G. Influence of tegaserod on proximal gastric tone and on the perception of gastric distention in functional dyspepsia. Neurogastroenterol Motil 2011;23:e32-e39.

16. Parkman HP, Carlson MR, Gonyer D. Metoclopramide nasal spray reduces symptoms of gastroparesis in women, but not men, with diabetes: results of a phase 2B randomized study. Clin Gastroenterol Hepatol 2015;13:1256-1263, e1.

17. McCallum RW, Cynshi O. Efficacy of mitemcinal, a motilin agonist, on gastrointestinal symptoms in patients with symptoms suggesting diabetic gastropathy: a randomized, multi-center, placebo-controlled trial. Aliment Pharmacol Ther 2007;26:107-116.

18. Oh JH, Pasricha PJ. Recent advances in the pathophysiology and treatment of gastroparesis. J Neurogastroenterol Motil 2013;19:18-24. 